\title{
Dynamic FDG-PET/CT in the Initial Staging of Primary Breast Cancer: Clinicopathological Correlations
}

\author{
Kornélia Kajáry $^{1} \cdot$ Zsolt Lengyel $^{1} \cdot$ Anna-Mária Tőkés $^{2} \cdot$ Janina Kulka $^{2} \cdot$ Magdolna Dank $^{3} \cdot$ Tímea Tőkés $^{3}$ (DD
}

Received: 8 January 2019 / Accepted: 13 March 2019 /Published online: 3 April 2019

(C) The Author(s) 2019

\begin{abstract}
Our aim was to evaluate correlation between clinicopathological features (clinical $\mathrm{T}$ and clinical $\mathrm{N}$ stages; histological type; nuclear grade; hormone-receptor and HER2 status, proliferation activity and tumor subtypes) of breast cancer and kinetic parameters measured by staging dynamic FDG-PET/CT examinations. Following ethical approval and patients' informed consent we included 34 patients with 35 primary breast cancers in our prospective study. We performed dynamic PET imaging, and assessed plasma activity noninvasively. To delineate primary tumors we applied a frame-by-frame semi-automatic softwarebased correction of motion artefacts. FDG two-compartment kinetic modelling was applied to assess K1, k2, k3 rate coefficients and to calculate $\mathrm{Ki}$ (tracer flux constant) and MRFDG (FDG metabolic rate). We found that k3, Ki and MRFDG were significantly higher in higher grade $(p=0.0246,0.0089$ and 0.0076 , respectively), progesterone-receptor negative $(p=0.0344,0.0217$ and 0.0132$)$ and highly-proliferating $(p=0.0414,0.0193$ and 0.0271$)$ tumors as well as in triple-negative and hormone-receptor negative/HER2-positive subtypes $(p=0.0310,0.0280$ and 0.0186$)$. Ki and MRFDG were significantly higher in estrogenreceptor negative tumors $(p=0.0300$ and 0.0247 , respectively). Ki was significantly higher in node-positive than in nodenegative disease $(p=0.0315)$. None of the assessed FDG-kinetic parameters showed significant correlation with stromal TIL. In conclusion, we confirmed a significant relationship between kinetic parameters measured by dynamic PET and the routinely assessed clinicopathological factors of breast cancer: high-grade, hormone-receptor negative tumors with high proliferation rate are characterized by higher cellular FDG-uptake and FDG-phosphorylation rate. Furthermore, we found that kinetic parameters based on the dynamic examinations are probably not influenced by stromal TIL infiltration.
\end{abstract}

Keywords Breast cancer $\cdot$ Kinetics $\cdot$ PET-CT $\cdot$ Tumor-infiltrating lymphocytes

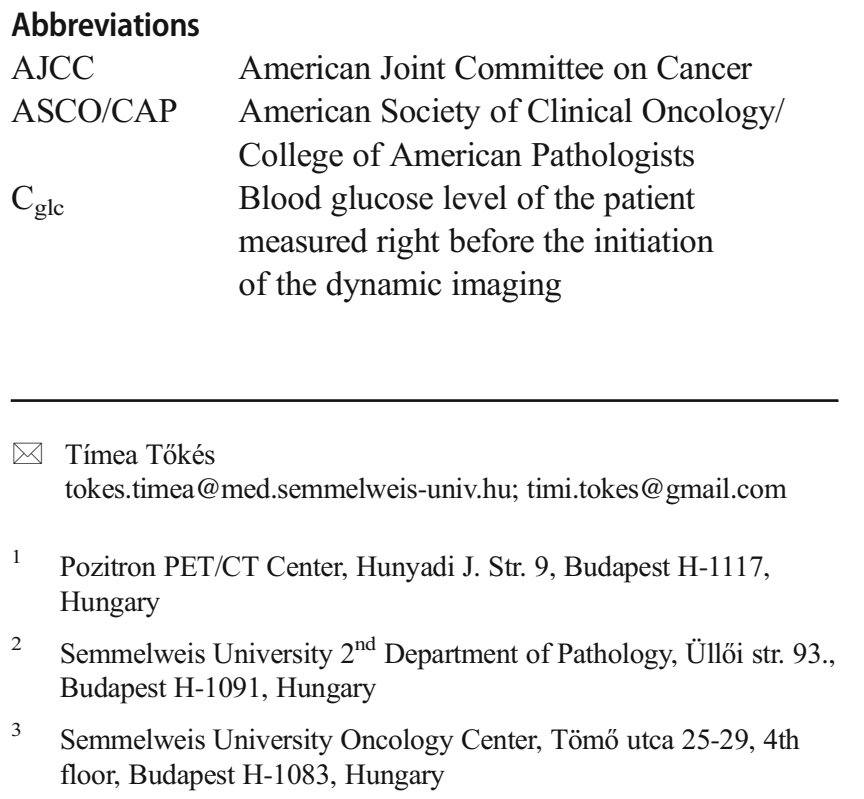

Abbreviations

American Joint Committee on Cancer

College of American Pathologists

Blood glucose level of the patient

measured right before the initiation

of the dynamic imaging

$\begin{array}{ll}\text { ER } & \begin{array}{l}\text { Oestrogen receptor } \\ \text { FDG-PET/CT }\end{array} \\ & \begin{array}{l}\text { 2-deoxy-2[18F]fluoro-D-glucose } \\ \text { positron emission tomography and } \\ \text { computer tomography }\end{array} \\ \text { FISH } & \begin{array}{l}\text { Fluorescent in situ hybridization } \\ \text { HER2 }\end{array} \\ \text { HR } & \begin{array}{l}\text { Human epidermal growth factor receptor } 2 \\ \text { and progesterone receptors })\end{array} \\ \text { HR-/ HER2+ } & \begin{array}{l}\text { Hormone-receptor negative and } \\ \text { HER2-positive; }\end{array} \\ \text { HR+/ HER2+ } & \begin{array}{l}\text { Hormone-receptor positive and } \\ \text { HER2-positive; }\end{array} \\ \text { HR+/ HER2+ } & \begin{array}{l}\text { Hormone-receptor positive } \\ \text { and HER2-negative. }\end{array} \\ \text { IBC NST } & \begin{array}{l}\text { Invasive breast carcinomas } \\ \text { of no special type } \\ \text { Immunohistochemistry } \\ \text { NHC }\end{array} \\ \text { Ki } & \text { Net tracer influx constant }\end{array}$




\begin{tabular}{|c|c|}
\hline Ki-67 LI & Ki-67 labelling index \\
\hline LIQ-UIQ & $\begin{array}{l}\text { Lower interquartile-upper } \\
\text { interquartile }\end{array}$ \\
\hline LV & Left ventricle \\
\hline MRFDG & $\begin{array}{l}\text { FDG metabolic rate in the tissue, } \\
\mu \mathrm{mol} / \mathrm{min} / 100 \mathrm{~g} \text { tissue }\end{array}$ \\
\hline OSEM & Ordered subsets expectation maximization \\
\hline PR & Progesterone receptor \\
\hline SD & Standard deviation \\
\hline SE-TUKEB & $\begin{array}{l}\text { Semmelweis University Institutional } \\
\text { Review Board }\end{array}$ \\
\hline SUV & Standardized Uptake Value \\
\hline TAC & Time-activity-curve \\
\hline TIL & Tumor infiltrating lymphocytes \\
\hline TNBC & Triple negative breast cancer \\
\hline VOI & Volume of interest \\
\hline
\end{tabular}

\section{Introduction}

In locally advanced breast cancer current guidelines suggest 2 deoxy-2[18F]fluoro-D-glucose positron emission tomography and computer tomography (FDG-PET/CT) as a useful and accurate tool for staging. Additionally, its role is also emerging in the response evaluation during neoadjuvant therapies [1]. Most commonly PET/CT scans are evaluated semi-quantitatively: the amount of the accumulated radiotracer is measured at one predefined time-point after injection (i.e. static imaging) and characterized by the calculated Standardized Uptake Value (SUV). SUV shows strong correlation with the clinicopathological characteristics of breast cancer, such as histological tumor type, biological subtype, grading and proliferation rate $[2$, 3]. Nonetheless, the static nature of SUV measurement can introduce several sources of bias, most importantly it is highly dependent on the time elapsed from tracer injection as well as body composition and glucose-metabolism of the patient [4]. These limitations of the SUV parameter could cause several bias during the staging and response evaluation. Therefore, in our current study we used dynamic PET imaging to accurately quantify the radiotracer uptake by the kinetic analysis of FDGaccumulation over a period of time.

In case of dynamic breast PET imaging, data collection lasted constantly for $60 \mathrm{~min}$, right directly after the tracer injection. The compartment modelling of FDG-distribution is used to evaluate the data acquired by dynamic imaging. Using the FDG-two-compartment model we could estimate the important rate parameters of the tracer flow between the blood-pool (first, plasma compartment) and the investigated tissue compartments (i.e. a transfer compartment, where FDG is intracellular and a metabolic compartment where FDG is in FDG-6-phosphate form). The estimated rate constants describe the influx and efflux of the FDG between these compartments: K1 and k2 are the rate constants representing carrier-mediated transport of FDG from plasma to tissue (K1) and back from tissue to plasma ( $\mathrm{k} 2)$, in the transfer compartment. $\mathrm{k} 3$ represents the rate constant for phosphorylation of the FDG by the hexokinase enzyme in the metabolic compartment. Based on the above listed parameters the net tracer influx constant $[\mathrm{Ki}=(\mathrm{K} 1 * \mathrm{k} 3) /$ $(\mathrm{k} 2+\mathrm{k} 3)]$ could be calculated. $[5,6]$.

We aimed to investigate the possible role of dynamic imaging in the characterization of breast cancer during the initial clinical staging. We assessed the correlations between the kinetic parameters measured by dynamic PET examinations (i.e. $\mathrm{K} 1, \mathrm{k} 2, \mathrm{k} 3, \mathrm{Ki}$ ) and the routinely used predictive and prognostic factors of breast cancers - such as clinical TNM, histological tumor type, tumor grade, proliferation rate and receptor status (i.e. hormone-receptor (HR) and human epidermal growth factor receptor 2 (HER2) expression). We also defined the clinical subtypes of the tumors, due to their important role in therapeutic choices in the daily practice [7]. Moreover, a novel pathological marker was also assessed in our study: the presence of tumor infiltrating lymphocytes (TIL) in the stroma of the tumors. Immune-cells are playing a central role in shaping of tumor microenvironment and presence of TILs proved to be a possible prognostic factor for favorable clinical outcome by itself [8]. Additionally, the lymphocyte predominant subgroups of triple negative tumors and hormone-receptor negative and HER2-positive (HR-/HER2+) tumors show a favorable response to systemic therapy, but, interestingly, this is not the case in hormone-receptor positive breast cancers. TIL could also have a future role to predict the effectiveness of immunotherapies [8]. In our study we tried to investigate if there is a connection between the presence of TILs and the measured FDG-influx during dynamic PET examinations.

In summary, in our current paper we present the results of our study with dynamic FDG-PET/CT imaging used in the staging of breast cancer. Our focus is on - over the routinely assessed clinicopathological characteristics of breast tumors the rarely investigated correlations between tumor phenotype and FDG-kinetics, with special emphasis on clinical subtypes and tumor microenvironment (characterized by stromal TILs). We also plan to extend our research to the response evaluation during neoadjuvant therapies.

\section{Patients and Methods}

\section{Patient Enrollment}

In our prospective study we included patients with invasive breast cancer. The inclusion criteria were the same as in our earlier published study [9], as follows: 1) diagnosis of primary breast cancer confirmed by core biopsy; (2) patients who were referred to the multidisciplinary tumor board of Semmelweis University Oncology Center in whom the board acknowledged the need of whole-body staging by using FDG-PET/ 
CT; (3) only tumors of at least $20 \mathrm{~mm}$ ( $\mathrm{T} 1 \mathrm{c} \leq$ ) were included to avoid the negative impact of partial volume effect in case of smaller lesions [10]; (4) patients with ECOG performance status 0 or 1 and age above 18 years; (5) signed written informed consent form.

Patients were excluded from the current analysis if: (1) any distant metastasis of parenchymal tissues was confirmed by staging FDG-PET/CT examinations (the presence of bone metastases was permitted); (2) chemotherapy was already initiated or radiation therapy was applied previously in the region of the affected breast; (3) inflammatory breast cancer; (4) pregnant or breast feeding patients; (5) claustrophobia; (6) patients who were not able or did not want to lie still for 60 min during the examination - especially with known co-morbidities which interfere with the need of prolonged immobility (Parkinson disease, musculoskeletal disorders etc.); (7) if a peripheral venous access could not be ensured. Only those patients can be considered for dynamic PET who are capable of successful cooperation during the whole, prolonged time of the imaging procedure.

Clinical TNM (cTNM stages) was assessed by using routinely applied diagnostic imaging modalities (X-ray and ultrasound mammography, and FDG-PET/CT) according to American Joint Committee on Cancer (AJCC) TNM 8th guideline [11].

Ethical approval for the study was given by the Semmelweis University Institutional Review Board (SETUKEB No.119/2013; date of approval: 24 June 2013). Every patient was informed personally and written informed consent form was signed by them before the enrollment.

\section{Histopathological Analysis}

Formalin-fixed, paraffin-embedded pre-treatment core biopsy samples were examined at the Semmelweis University 2nd Department of Pathology, by board-certified pathologists. Detailed histological characterization was performed on the core biopsy samples, including the determination of histological type and nuclear grade. Immunohistochemistry (IHC) was routinely performed to evaluate hormone receptor - estrogen (ER) and progesterone (PR) - status, as well as HER2 expression according to international guidelines, with the evaluations of tumor proliferation by using the $\mathrm{Ki}-67$ labeling index (Ki-67 LI).

Hormone receptor positivity was confirmed if Allred score was above or equal to 3 [12]. HER2 overexpression was defined as IHC $3+$. HER2 $1+$ or 0 tumors were considered HER2 negative. For IHC 2+ samples, fluorescent in situ hybridization (FISH) was performed to confirm gene amplification. HER2 status was defined according to the American Society of Clinical Oncology/College of American Pathologists (ASCO/CAP) Guideline valid at the time of diagnosis, i.e. HER2-positive patients treated between January 2008 and
November 2013 were identified according to the 2007 ASCO/CAP Guideline [13] and from then on according to the Guideline published in October 2013 [14]. Ki-67 LI was scored as described by Dowsett et al. [15, 16]: every slide was assessed visually and the proportion of positive cells was determined by counting approximately 500 tumor cells at $400 \mathrm{x}$ magnification. For Ki-67 LI a cell was considered positive if any nuclear signal was observed, similar to the earlier published studies of Tökés et al. [17].

Biological subtype of the tumors was defined according to the recommendations of the 2017 St. Gallen International Breast Cancer Conference [7]. Triple negative (TNBC), hormone-receptor negative and HER2-positive (HR-/HER2+ ), hormone-receptor positive and HER2-positive (HR+/ HER2+) and hormone-receptor positive and HER2-negative (HR+/HER2-) tumors were separated based on the expression of ER, PR and HER2, taking into account the Ki-67 LI status, as well.

Additionally, the core-biopsy samples were also evaluated for the presence of tumor infiltrating lymphocytes (TIL) in the stroma of the tumors, according to the TILs Working Group guideline published in 2015 [8]. Evaluation of the stromal TILs was performed by co-authors TAM and JK, experts of the field.

\section{PET/CT Studies}

All PET examinations were performed with Siemens Biograph TruePoint 6 HD (Siemens Molecular Imaging Division, Knoxville, USA) PET/CT scanner. Patients were prepared according to standard guidelines $[18,19]$. Most importantly, the examinations were preceded by at least 6hour starvation. The level of blood glucose and body-weight were measured upon arrival to the PET/CT Center. The average weight of the investigated patients $(n=34)$ was $67.7 \pm$ $12.5 \mathrm{~kg}$ and the mean blood glucose was $5.1 \pm 0.9 \mathrm{mmol} / \mathrm{L}$. The scans were acquired after the intravenous injection of 155.4-384.8 MBq (4.2-10.4 mCi) FDG according to body weight $(\sim 3.7 \mathrm{MBq} / \mathrm{kg} ; \sim 0.1 \mathrm{mCi} / \mathrm{kg})$. The mean dose of administered FDG was $254.6 \pm 51.9 \mathrm{MBq}(6.8 \pm 1.4 \mathrm{MBq})$. Intravenous injection of tracer was performed with the patient lying on the examination table (minute zero). PET data collection was continuous (list mode) from minute zero, with fixed bed position over the chest region ( $\mathrm{Z}$ axis length: $21.6 \mathrm{~cm}$ ). Dynamic PET data acquisition lasted for $60 \mathrm{~min}$, with the patient lying still on the examination table of the $\mathrm{PET} / \mathrm{CT}$ scanner. The dynamic imaging sequence, starting with the initiation of the bolus was $8 \times 15$ seconds, $8 \times 30$ seconds, $2 \times 60$ seconds, $3 \times 2$ minutes, $4 \times 3$ minutes, $1 \times 4$ minutes and $6 \times 5$ minutes. PET images were corrected for decay, attenuation and normalization, scatter and randoms rejection, as well as for dead time. PET data were acquired in three-dimensional mode and were reconstructed using CT 
data for attenuation correction with ordered subsets expectation maximization (OSEM) algorithm (4 iterations, 8 subsets), using a $168 \times 168 \times 74$ image matrix with voxel dimension of $4.07 \times 4.07 \times 3.0 \mathrm{~mm}$. Image reconstruction was performed as described earlier in our previous study [9]: the early frames of every dynamic study (from 1 to 4 , from 5 to 8 , from 9 to 10 and from 11 to 12 frames, respectively) was averaged in a time-weighted manner (which process is offered by most of the available software tools) to correct the earlier described inaccuracies without significant degradation of time resolution.

Collected dynamic data were analyzed by using PMOD software (v3.310, Zürich, Switzerland). Every study was assessed by the same two experienced observers (KK and TT). To generate the time-activity-curves (TACs), changes of activity concentrations were assessed in the tumor and in the blood pool (i.e. plasma). To assess plasma activity, observers draw a sphere volume of interest (VOI) in the left ventricle (LV) of the heart sized $15 \mathrm{~mm}$ in diameter. Similarly, $15 \mathrm{~mm}$ sized sphere VOIs were also used to measure the primary tumors' activity over time. To eliminate movement artefacts due to involuntary movements and breathing, positions of the VOIs were corrected by using a semi-automatic, computer-assisted method, published earlier [9]: at first we placed a VOI over the tumor, which exceeded the longest diameter of the examined lesion with at least $10 \mathrm{~mm}$, and then we centered the VOI to the voxel with the highest uptake in the target region. By reducing the size of the VOI to $15 \mathrm{~mm}$ in diameter, we finalized the motion correction of the current time-frame. This procedure was repeated on every time-frame of the dynamic studies. This approach seemed to be reliable, reproducible, with low inter-observer variability, compared to manual motion correction [9].

Thereafter TACs were defined by using the VOIs of left ventricle and the tumor(s), afterwards FDG two-compartment kinetic modelling (non-reversible, $\mathrm{k} 4=0$ ) was applied to assess K1, k2, k3 coefficients and to calculate Ki and MRFDG (the FDG metabolic rate in the tissue, $\mu \mathrm{mol} / \mathrm{min} / 100 \mathrm{~g}$ tissue). Relationship of these parameters is defined by the following equation:

$\mathrm{MRFDG}=\mathrm{C}_{\mathrm{glc}} \times \mathrm{K}_{\mathrm{i}}$

where $\mathrm{C}_{\mathrm{glc}}$ is the blood glucose level of the patient measured right before the initiation of the dynamic PET imaging. It should be highlighted that MRFDG is not equivalent to the glucose metabolic rate (referred as MRGlu), since the constant which describes the relationship between the FDG accumulation and glucose consumption (i.e. the lumped constant) is not defined yet in case of breast tissues - therefore the exact rate of the glucose metabolism cannot be calculated reliably $[5,20]$.

\section{Statistical Analysis}

Data were expressed as mean \pm standard deviation (SD) or median and lower interquartile-upper interquartile (LIQUIQ). All applied statistical tests were two-sided and $p$ values $<0.05$ were considered significant. Shapiro-Wilks test was applied to test the normality of the data. Connections between clinicopathological characteristics and the kinetic parameters were assessed by using Mann-Whitney and KruskalWallis tests.

For data collection and processing we used Microsoft Excel 2010 (Microsoft Corporation, Redmond Washington, USA), Statistica v13.2 (StatSoft Inc.) and SPSS v22.0 (IBM Corporation) software.

\section{Results}

A total of 35 lesions detected during 34 dynamic studies were included in this current analysis. Dominantly grade $3(19 / 35$; $54 \%$ ) invasive breast carcinomas of no special type (IBC NST) $(30 / 35 ; 85.7 \%)$ were evaluated. 24 of the included tumors were ER positive $(69 \%)$ and 22 were PR positive $(63 \%)$ (total number of hormone receptor positive $(\mathrm{HR}+$ ) lesions was 25 - one lesion showed solely PR positivity, but ER negativity). Only six tumors showed HER2 positivity (17\%) and 27 tumors showed elevated proliferation activity, i.e. Ki-67 LI > $20 \%(77 \%)$. (Table 1).

Regarding clinical subtypes of the investigated lesions, eight tumors were TNBC (22.9\%) and 2 were HR-/HER2+ $(5.7 \%) .4$ tumors were HR+/HER2+ (11.4\%). The remaining 21 lesions were HR+/HER2-. Amongst these, five were luminal A-like tumor (14.3\%), seven were luminal B-like (20\%) and 9 lesions belonged to the intermediate subgroup (25.7\%).

We analyzed the relationship between the kinetic parameters measured on the dynamic PET/CTs and the known predictive and prognostic factors (i.e. clinical $\mathrm{T}$ and clinical $\mathrm{N}$ stages; histological type; nuclear grade; ER, PR and HER2 status, Ki-67 LI and tumor subtype) in pre-therapy core-biopsies (Table 2).

We did not find significant differences according to tumor size (clinical T categories). However, in case of nodal involvement Ki was significantly higher in node positive than in node negative disease $(p=0.0315)$.

Tumors with higher nuclear grade showed significantly higher k3 $(p=0.0246), \mathrm{Ki}(p=0.0089)$ and MRFDG $(p=0.0076)$.

Regarding hormone receptor status, we found that $\mathrm{Ki}(p=$ 0.0300 and $p=0.0217$, respectively) and MRFDG ( $p=$ 0.0247 and $p=0.0132$, respectively) were significantly higher in ER negative and PR negative tumors than in hormone receptor positive $(\mathrm{HR}+)$ cancers. In PR negative tumors, $\mathrm{k} 3$ was also significantly higher compared to PR positive lesions ( $p=$ $0.0344)$. HER 2 status did not show any correlation with the kinetic parameters. Tumors with elevated Ki-67 LI (i.e. high 
Table 1 Clinicopathological characteristics $(n=34$ patients with $n=35$ lesions)

\begin{tabular}{|c|c|c|}
\hline Clinical TNM ( $n=34$ patients) & No. & $\%$ \\
\hline \multicolumn{3}{|l|}{ Clinical T stage } \\
\hline cT1c & 4 & 11.8 \\
\hline cT2 & 25 & 73.5 \\
\hline $\mathrm{cT} 3$ & 5 & 14.7 \\
\hline \multicolumn{3}{|l|}{ Clinical N stage } \\
\hline $\mathrm{cNO}$ & 9 & 26.5 \\
\hline $\mathrm{cN}$ positivity $(\mathrm{cN} 1-3)$ & 25 & 73.5 \\
\hline \multicolumn{3}{|l|}{ Clinical M stage } \\
\hline $\mathrm{cM} 0$ & 33 & 97 \\
\hline $\mathrm{cM} 1 *$ & 1 & 3 \\
\hline Pathological Characteristics ( $n=35$ lesions) & No. & $\%$ \\
\hline \multicolumn{3}{|l|}{ Histology } \\
\hline IBC-NST & 30 & 85.7 \\
\hline Invasive lobular carcinoma & 2 & 5.7 \\
\hline Other $\dagger$ & 3 & 8.6 \\
\hline \multicolumn{3}{|l|}{ Grade $\$$} \\
\hline 1 & 2 & 5.7 \\
\hline 2 & 8 & 22.9 \\
\hline 3 & 19 & 54.3 \\
\hline \multicolumn{3}{|l|}{ Estrogen receptor status } \\
\hline ER positive & 24 & 68.6 \\
\hline ER negative & 11 & 31.4 \\
\hline \multicolumn{3}{|l|}{ Progesterone receptor status } \\
\hline PR positive & 22 & 62.9 \\
\hline PR negative & 13 & 37.1 \\
\hline \multicolumn{3}{|l|}{ HER2 status } \\
\hline HER2 positive & 6 & 17.1 \\
\hline HER2 negative & 29 & 82.9 \\
\hline \multicolumn{3}{|l|}{ Proliferation rate } \\
\hline High Ki-67 LI & 27 & 77.1 \\
\hline Low Ki-67 LI & 8 & 22.9 \\
\hline \multicolumn{3}{|l|}{ Clinical subtypes\# } \\
\hline Triple negative (TNBC) & 8 & 22.9 \\
\hline HR-/HER2+ & 2 & 5.7 \\
\hline HR+/HER2+ & 4 & 11.4 \\
\hline HR+/HER2-: Luminal A-like tumors & 5 & 14.3 \\
\hline HR+/HER2-: Intermedier subgroup & 9 & 20 \\
\hline HR+/HER2-: Luminal B-like tumors & 7 & 25.7 \\
\hline
\end{tabular}

*1 patient was included with bone metastases on the initial staging †two invasive pleiomorph carcinoma, one undifferentiated adenocarcinoma of the breast

\$in 6 patients tumor grade is unknown . \# 1 patient has a PR-positive but ER-negative tumor, therefore the overall number of $\mathrm{HR}+$ lesions is 25

Abbreviations: IBC NST invasive breast carcinomas of no special type, $E R$ estrogen receptor, $P R$ progesterone receptor, $H E R 2$ human epidermal growth factor receptor 2, Ki-67 LI Ki-67 labeling index, HR-/HER2+ hormone-receptor negative and HER2-positive, HR+/HER2+ hormonereceptor positive and HER2-positive, $H R+/ H e r 2$ - hormone-receptor positive and HER2-negative proliferation activity) showed significantly higher $\mathrm{k} 3$ ( $p=$ $0.0414), \mathrm{Ki}(p=0.0193)$ and MRFDG $(p=0.0271)$.

Additionally, FDG-kinetics were also compared between the St. Gallen 2017 consensus [7] based clinical subtypes. Initially, we did not find significant differences between the clinical subtype distribution of the tumors and the investigated kinetic parameters. However, considering the low number of cases present in each clinical subtype, we performed an additional analysis: subgroups were compared based on their prognostic significance - more aggressive TNBC and HR-/HER2+ subtypes $(n=10)$ were compared with hormone-receptor positive lesions (HR+/HER2+ and HR+/HER2- tumors, $n=25)$. TNBC and HR-/HER2+ subtypes showed significantly higher k3 $(p=0.0310), \mathrm{Ki}(p=0.0280)$ and MRFDG $(p=0.0186)$ compared to hormone-receptor positive diseases.

In summary, three kinetic parameters $(\mathrm{k} 3, \mathrm{Ki}$ and MRFDG) were strongly associated with the biological behavior of the tumors. The correlations between $\mathrm{k} 3, \mathrm{Ki}$ and MRFDG and tumor grade, ER and PR status, Ki-67 LI and subtype groups are presented on Figs. 1, 2, and 3. All of these correlations were significant except the correlation between $\mathrm{k} 3$ and ER status.

We also analyzed the correlations between the presence of stromal TILs and kinetic parameters of the primary tumors. Data about the presence TIL was available in 23 patients. Median TIL was 5\% (LIQ-UIQ:1-7.5\%), most of the lesions showed slightly elevated but not significantly high stromal TIL content (12/23 lesions showed TIL between $1 \%$ and $20 \%$ ). Every investigated kinetic parameter was higher in tumors with TIL infiltration $>1 \%$ and higher $\mathrm{K} 1$ and $\mathrm{k} 2$ was measured in tumors with increased TIL $(>20 \%)$, but none of these observations were statistically significant (Table 2).

\section{Discussion}

In breast cancer, the routinely used predictive and prognostic (bio)markers are mainly based on invasive tumor sampling and pathological assessment (i.e. tumor type, grade, proliferation activity, receptor status etc.). The relationship between these markers and FDG-avidity measured during static FDGPET/CT imaging is already known and substantially investigated [2, 3]. Amongst others, our research group already highlighted the strong relationship between SUV and several clinicopathological factors of breast cancer: lesions with IBCNST phenotype showing high-grade features and increased proliferation, with triple negativity and axillary lymph node positivity usually show higher FDG-uptake compared to lowgrade, luminal A-like tumors with node negativity [2, 3].

On the contrary, the role of dynamic PET imaging is under debate in breast cancer. The indisputable advantage of dynamic FDG-PET/CT is its capability to accurately quantify FDGuptake. Therefore, dynamic imaging carries a potential to 
Table 2 Relationship between the clinicopathological characteristics of the investigated breast tumors and the kinetic parameters of the performed dynamic PET examinations

\begin{tabular}{|c|c|c|c|c|c|}
\hline Feature (No. of tumors) & $\begin{array}{l}\text { K1 } \\
\text { mean }\end{array}$ & $\begin{array}{l}\mathrm{k} 2 \\
\text { mean }\end{array}$ & $\begin{array}{l}\mathrm{k} 3 \\
\text { mean }\end{array}$ & $\begin{array}{l}\mathrm{Ki} \\
\text { mean }\end{array}$ & $\begin{array}{l}\text { MRFDG } \\
\text { mean }\end{array}$ \\
\hline \multicolumn{6}{|l|}{ Clinical T stage } \\
\hline $\mathrm{T} 1 \mathrm{c}(4)$ & 0.185 & 0.543 & 0.062 & 0.018 & 8.701 \\
\hline $\mathrm{T} 2(26)$ & 0.375 & 1.095 & 0.099 & 0.036 & 17.531 \\
\hline $\mathrm{T} 3(5)$ & 0.258 & 0.779 & 0.164 & 0.044 & 23.292 \\
\hline$p$ value & 0.3742 & 0.9003 & 0.2206 & 0.0834 & 0.0780 \\
\hline \multicolumn{6}{|l|}{ Clinical N stage } \\
\hline N0 (9) & 0.292 & 0.909 & 0.076 & 0.023 & 12.961 \\
\hline $\mathrm{N} 1-3(26)$ & 0.352 & 1.014 & 0.114 & 0.039 & 18.862 \\
\hline$p$ value & 0.5459 & 0.2271 & 0.2575 & 0.0315 & 0.1046 \\
\hline \multicolumn{6}{|l|}{ Histology $*$} \\
\hline IBC-NST (30) & 0.337 & 1.004 & 0.101 & 0.034 & 16.951 \\
\hline other (5) & 0.333 & 0.884 & 0.125 & 0.041 & 19.709 \\
\hline \multicolumn{6}{|l|}{ Nuclear grade $\dagger$} \\
\hline $1(2)$ & 0.149 & 0.427 & 0.049 & 0.015 & 6.148 \\
\hline $2(8)$ & 0.353 & 0.983 & 0.071 & 0.022 & 10.838 \\
\hline $3(19)$ & 0.371 & 1.157 & 0.129 & 0.042 & 21.875 \\
\hline$p$ value & 0.3075 & 0.3417 & 0.0246 & 0.0089 & 0.0076 \\
\hline \multicolumn{6}{|l|}{ ER status } \\
\hline positive (24) & 0.279 & 0.727 & 0.094 & 0.032 & 14.934 \\
\hline negative (11) & 0.462 & 1.555 & 0.128 & 0.041 & 22.604 \\
\hline$p$ value & 0.4095 & 0.5872 & 0.0667 & 0.0300 & 0.0247 \\
\hline \multicolumn{6}{|l|}{ PR status } \\
\hline positive (22) & 0.277 & 0.735 & 0.088 & 0.029 & 13.775 \\
\hline negative (13) & 0.436 & 1.414 & 0.132 & 0.044 & 23.386 \\
\hline$p$ value & 0.2035 & 0.5782 & 0.0344 & 0.0217 & 0.0132 \\
\hline \multicolumn{6}{|l|}{ HER2 status } \\
\hline positive (6) & 0.369 & 1.136 & 0.104 & 0.033 & 15.824 \\
\hline negative (29) & 0.329 & 0.956 & 0.105 & 0.035 & 17.659 \\
\hline$p$ value & 0.5933 & 0.2184 & 0.4801 & 0.8814 & 0.9151 \\
\hline \multicolumn{6}{|l|}{ Ki-67 LI } \\
\hline low (8) & 0.269 & 0.802 & 0.062 & 0.021 & 9.931 \\
\hline $\operatorname{high}(27)$ & 0.356 & 1.042 & 0.117 & 0.039 & 19.542 \\
\hline$p$ value & 0.8323 & 0.9843 & 0.0414 & 0.0193 & 0.0271 \\
\hline \multicolumn{6}{|l|}{ Subtype } \\
\hline Triple negative (8) & 0.456 & 1.602 & 0.134 & 0.043 & 23.844 \\
\hline HR-/HER2+ (2) & 0.674 & 2.087 & 0.136 & 0.041 & 21.863 \\
\hline HR+/HER2+ (4) & 0.217 & 0.661 & 0.088 & 0.029 & 12.804 \\
\hline HR+/HER2- (21) & 0.281 & 0.710 & 0.093 & 0.032 & 15.303 \\
\hline$p$ value (all) & 0.1932 & 0.2396 & 0.1666 & 0.1865 & 0.1374 \\
\hline$p$ value (TNBC and HER $2+$ vs. all $\mathrm{HR}+$ ) & 0.1514 & 0.2703 & 0.0310 & 0.0280 & $\underline{0.0186}$ \\
\hline \multicolumn{6}{|l|}{ TILt } \\
\hline $\mathrm{TIL} \leq 1 \%(8)$ & 0.225 & 0.483 & 0.089 & 0.033 & 15.346 \\
\hline $1 \%<\mathrm{TIL}<20 \%(12)$ & 0.347 & 1.023 & 0.109 & 0.036 & 18.807 \\
\hline $\mathrm{TIL} \geq 20 \%$ & 0.742 & 2.916 & 0.095 & 0.032 & 16.048 \\
\hline$p$ value (all) & 0.4713 & 0.0940 & 0.2055 & 0.5849 & 0.4346 \\
\hline
\end{tabular}

$p$ values: in bold. Underlined: statistically significant difference

*Due to low case numbers and statistical power we did not perform a statistical comparison;

$\dagger$ Missing in case of 6 tumors; $\$$ missing in case of 12 tumors

Abbreviations: IBC NST invasive breast carcinomas of no special type, ER estrogen receptor, $P R$ progesterone receptor, HER 2 human epidermal growth factor receptor 2, Ki-67 LI Ki-67 labeling index, TNBC triple negative, HR-/ HER2+ hormone-receptor negative and HER2-positive, HR+/ HER2+ hormone-receptor positive and HER2-positive, $H R+/ H E R 2+$ hormone-receptor positive and HER2-negative, TIL tumor infiltrating lymphocytes

define novel predictive and prognostic biomarkers in vivo for numerous tumor types. The assessment of the correlations between in vivo measured parameters of FDG tracer kinetics and biological behavior of breast tumors can increase the value of dynamic PET/CT imaging in the daily practice.
However, implementation of dynamic PET imaging is complicated, time- and resource-consuming; therefore, the delineation of a suitable patient population is essential. A few studies already investigated the role of the parameters derived from dynamic data collection followed by tracer kinetic 

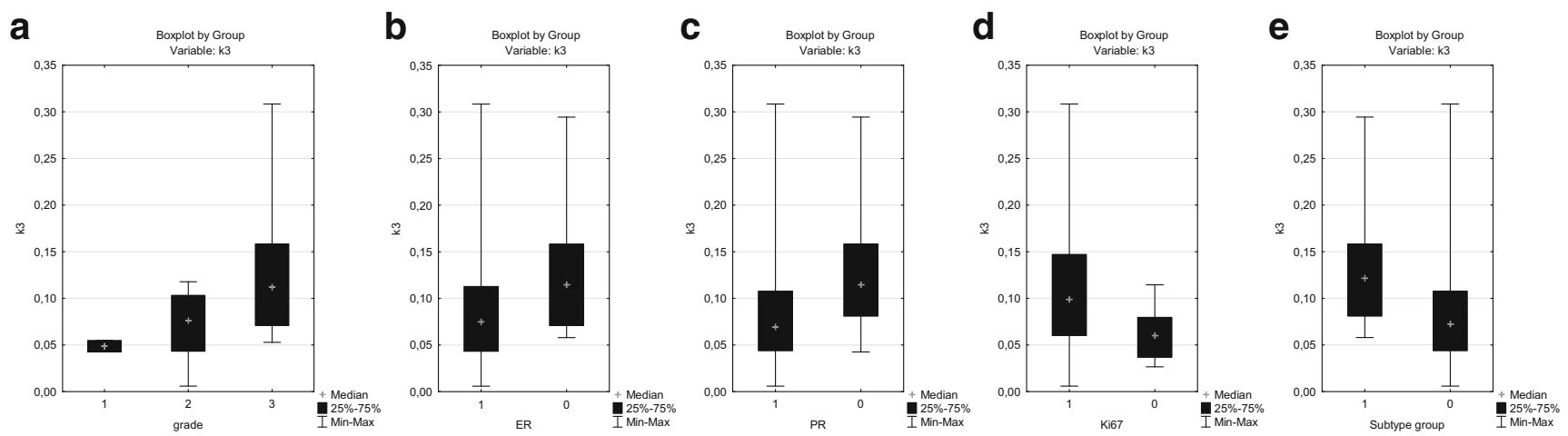

Fig. 1 Box-plot figures representing the comparison of $\mathrm{k} 3$ values between (a) grade 1, grade 2 and grade 3 tumors ( $p=0.0246)$; (b) estrogen (ER) positive $(+)$ and ER negative $(-)$ tumors $(p=0.0667)$; (c) progesterone (PR) positive $(+)$ and PR negative $(-)$ tumors $(p=0.0344)$; (d) tumors with high and low Ki-67 LI $(p=0.0414)$. We also compared the value of $\mathrm{k} 3$ between two patient groups based on biological subtypes (e): the more aggressive TNBC and HR-/HER2+ subtypes(group 1) and the hormone-receptor positive lesions (HR+/HER2+ and HR+/HER2tumors) (group 2) $(p=0.0310)$ modelling in the staging of breast cancer [21] as well as during response evaluation to neoadjuvant therapy [20-27]. Our study focused on the rarely investigated correlations between tumor phenotype and FDG-kinetics, with special emphasis on clinical subtypes and tumor microenvironment (characterized by stromal TILs), which has gained special attention in recent years of cancer research.

In our study, while analyzing core-biopsy samples of breast cancer, we did not find any correlation between lesion size and the kinetic parameters of the dynamic PET studies. If axillary lymph node status was positive per clinical-imaging investigations ( $>\mathrm{cN} 0$ ), every measured kinetic parameter was higher compared to clinically node-negative ( $\mathrm{cN} 0)$ cases, but the relationship was only significant in case of Ki. Dunnwald et al. described similar results in their earlier published study regarding $\mathrm{Ki}$, but they found that $\mathrm{K} 1$ is also significantly higher in node positive disease (nonetheless, they did not published any results particularly about the correlations with $\mathrm{k} 2$ and $\mathrm{k} 3$ ) [21].
Regarding the biological characteristics of the assessed breast cancers, we found that every assessed kinetic parameter was elevated in high-grade tumors and this correlation was statistically significant in case of k3, Ki and MRFDG. When assessing the correlation with tumor proliferation, similar correlations were found: lesions with high Ki-67 LI showed significantly higher values of $\mathrm{k} 3, \mathrm{Ki}$ and MRFDG compared to lesions characterized by low Ki-67 LI. Dunnwald et al. found the same significant correlations for $\mathrm{Ki}$. They also assessed the relationship between Ki-67 LI and K1, but did not find a significant correlation with tumor grade or proliferation (it is note that they did not evaluate the role of k2 nor k3) [21].

Regarding hormone receptor status, significantly different FDG-kinetic parameters were detected in HR- tumors compared to HR+ lesions. In case of ER expression, $\mathrm{Ki}$ and MRFDG were significantly higher in ER-negative than in ER-positive lesions. Regarding PR the k3, Ki and MRFDG was significantly higher in PR-negative than in PR-positive
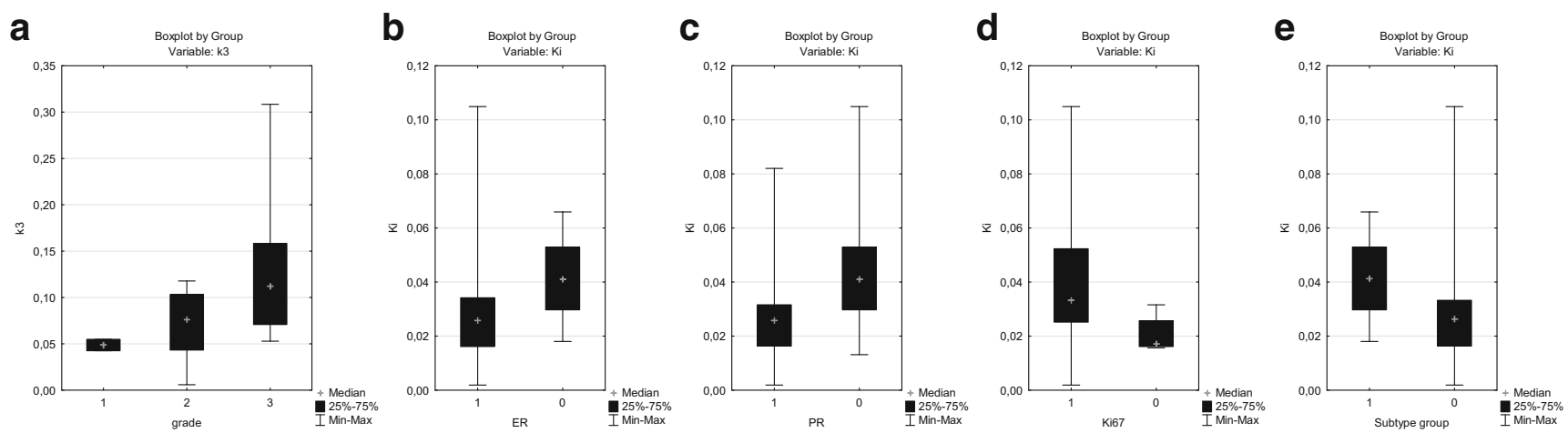

Fig. 2 Box-plot figures representing the comparison of $\mathrm{Ki}$ values between (a) grade 1, grade 2 and grade 3 tumors ( $p=0.0089$ ); (b) estrogen (ER) positive $(+)$ and ER negative $(-)$ tumors $(p=0.0300)$; (c) progesterone (PR) positive $(+)$ and PR negative $(-)$ tumors $(p=0.0217)$; (d) tumors with high and low Ki-67 LI $(p=0.0193)$. We also compared the value of Ki between two patient groups based on biological subtypes (e): the more aggressive TNBC and HR-/HER2+ subtypes(group 1) and the hormone-receptor positive lesions (HR+/HER2+ and HR+/HER2tumors) (group 2) ( $p=0.0028)$ 

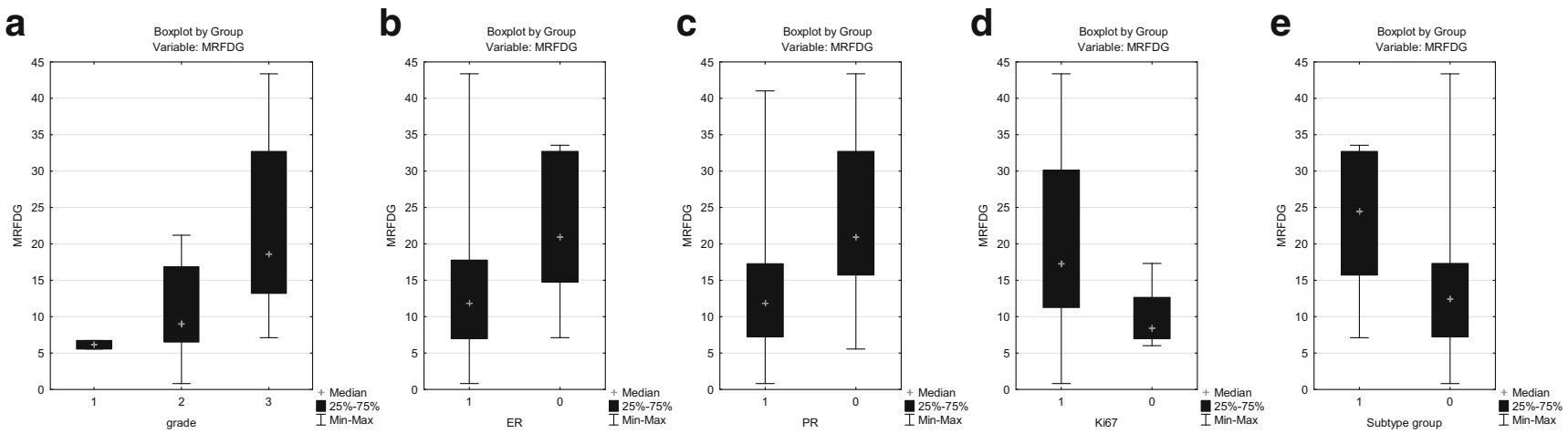

Fig. 3 Box-plot figures representing the comparison of MRFDG values between (a) grade 1, grade 2 and grade 3 tumors $(p=0.0076$ ); (b) estrogen (ER) positive $(+)$ and ER negative $(-)$ tumors $(p=0.0247)$; (c) progesterone (PR) positive $(+)$ and PR negative $(-)$ tumors $(p=0.0132)$; (d) tumors with high and low Ki-67 LI ( $\mathrm{p}=0.0271)$. We also compared the value of
MRFDG between two patient groups based on biological subtypes (e): the more aggressive TNBC and HR-/HER2+ subtypes(group 1) and the hormone-receptor positive lesions (HR+/HER2+ and HR+/HER2- tumors) (group 2) $(p=0.0186)$ tumors. In our study, we did not find a significant correlation between HER 2 positivity and the investigated kinetic parameters of the dynamic studies. Nonetheless, the group of TNBC and HR-/HER2+ subtypes showed significantly higher values of $\mathrm{k} 3, \mathrm{Ki}$ and MRFDG in comparison to hormone-receptor positive breast cancers (i.e. HR+/HER2+ and HR+/HER2tumors). Regarding hormone-receptor and HER2-status similar correlations were published earlier by Dunnwald et al. as was observed in our study, but only in case of $\mathrm{Ki}$ [21]. The analysis of the relationship between breast cancer subtypes and FDG-kinetics was not performed earlier, only in case of static PET/CT imaging, where similar correlations were described $[2,3]$. In our study TNBC and HR-/HER2+ subtypes which are characterized by worse clinical outcome compared to hormone-receptor positive $(\mathrm{HR}+)$ tumors - are confirmed to have high FDG-influx and a more prominent FDG metabolic rate, which explains the significantly higher SUVs of these phenotypes during routine static PET imaging [2, 3].

We also assessed the ratio of stromal TILs in the primary tumors, due to its possible prognostic and predictive role in breast cancer [8]. It is reasonable to hypothesize that tumor microenvironment - especially the amount of stromal TILs could affect the FDG uptake and distribution of malignant lesions. In gastric cancer [28] and in case of non-small cell lung cancer [29] a positive correlation was already described between the presence of TILs and FDG-uptake in case of routinely used static FDG-PET/CT imaging. Regarding breast cancer, this question was only assessed indirectly, in the recently published study of Fujii et al. [30], who found a relationship between higher neutrophil granulocyte/lymphocyte ratio (i.e. a marker of systemic inflammation) and higher-FDG uptake (also evaluated during static imaging). Nevertheless, in our study we did not find significant difference in the kinetic parameters of the dynamic studies when compared breast tumors with different ratios of stromal TILs. Our preliminary results suggest that the presence of stromal TILs did not affect significantly the measured FDG-kinetics of the dynamic studies in breast cancer patients - however this hypothesis must be further investigated by repeating our study in a larger group of patients.

Based on the above, we can highlight that the same correlations can be found between the kinetic parameters of the dynamic PET examinations and breast tumors' phenotype as were described earlier when only static FDG-PET/CT imaging was used in the staging of breast cancers [2, 3]. During dynamic imaging high-grade, hormone-receptor negative tumors with high proliferation rate are characterized by higher cellular FDG-uptake and FDG-phosphorylation rate (in harmony with earlier published results [21]). These lesions are showing significantly higher SUVs if static FDG-imaging is applied for staging [2,3]. Nevertheless, these tumors frequently present as locally advanced diseases with aggressive clinical behavior, therefore are regularly treated with neoadjuvant therapy. In this case, the limitations of the SUV parameter of static imaging could cause several bias during the response evaluation. For example if the patient lost significant bodyweight during the therapeutic course or had altered renal clearance due to the applied chemotherapies the value of the SUV could be diverged accordingly [4]. Dynamic imaging however is capable to accurately quantify the FDG-uptake and it is not affected by these bias. For this reason, testing of dynamic PET has already been begun in this specific indication [20-27]. Based on these earlier studies we can conclude that an initially high rate of FDG-influx and lower MRFDG as well as a lower post-therapeutic $\mathrm{Ki}$ and $\mathrm{K} 1$ are both predictive to favorable tumor response to the neoadjuvant therapy [21-25]. These result are probably related to the strong correlations between the value of $\mathrm{Ki}$ and tumor vascularization [23], blood flow [24] and functional tumor volume [26], which parameters are suitable to delineate residual disease, if still onset after the neoadjuvant treatment of breast tumors. Moreover, it 
seems likely that response evaluation with the kinetic parameters of the dynamic studies is not depending on the initial pretherapy FDG-uptake as much as the static evaluations (using the SUV) is. With dynamic imaging the follow-up of less FDG-avid breast tumors, with lower pre-therapy FDG-uptake can also be implemented [21, 22, 27].

Thus, based on our results, in the staging of breast cancer dynamic imaging may not have an outstanding added value due to its similar correlations with the clinico-pathological characteristics of the tumors which has already been described by static imaging studies. However, our current results are suggesting that if tumor microenvironment is taken into account, it seems likely that the parameters of dynamic imaging are not affected by TIL ratio as much as the routinely applied static imaging. Additionally, based on earlier preclinical and clinical studies, if neoadjuvant chemotherapy is planned in locally advanced disease, dynamic imaging could be a promising tool for response evaluation, by quantifying FDG-uptake more precisely when initial staging and post-therapy imaging is compared. Our next studies are addressed to further enlighten its role in the clinical routine.

The main limitations of our study is our VOI delineation method: the reported kinetic parameters only describing a $15 \mathrm{~mm}$ sized portion of the investigated tumors instead of the whole, sometimes heterogeneous tumor mass. Additionally, during motion correction the VOIs were centered on the most metabolically active part of the lesions, which could also be misleading, especially in large lesions. Additionally, the small number of tumors in which TIL was determined (only in $65 \%$ of the included patients) is also limiting the value of our results. To further clarify the correlation between tumor microenvironment and FDG-kinetics, our study must be repeated in larger group of patients.

In conclusion, our preliminary findings proved a significant relationship between kinetic parameters measured by dynamic PET and the routinely assessed clinicopathological factors, similarly as was known in case of static SUV measurements. We also confirmed that high-grade, hormone-receptor negative tumors with high proliferation rate are characterized by higher cellular FDG uptake and FDG phosphorylation rate based on dynamic imaging. Additionally, we further underlined the aggressive biological behavior of TNBC and HR-/HER2+ subtypes. Furthermore, we described that kinetic parameters measured by dynamic PET examinations are probably not influenced by stromal TIL infiltration.

\footnotetext{
Acknowledgements The entire research group would like to express their gratitude to the technicians of Pozitron PET/CT Center for their professional assistance in the implementation of the dynamic PET/CT studies. Authors thank Álmos Szentpéteri, multidisciplinary tumor board administrator and László Torgyík MD, deputy director of the Oncology Center of Semmelweis University as well as Tamás Györke MD PhD, director of the Nuclear Medicine Center of Semmelweis University for their valuable support during the patient selection of our study.
}

Funding Open access funding provided by Semmelweis University (SE). This research did not receive any specific grant from funding agencies in the public, commercial, or not-for-profit sectors. Dynamic PET/CT studies were financed by Pozitron PET/CT Center, workplace of two of the authors (KK and ZL).

Data Availability The datasets generated during and/or analysed during the current study are available from the corresponding author on reasonable reques.

\section{Compliance with Ethical Standards}

Conflict of Interest The authors declare that they have no conflict of interest.

Ethical Approval All procedures performed in studies involving human participants were in accordance with the ethical standards of the institutional and/or national research committee and with the 1964 Helsinki declaration and its later amendments or comparable ethical standards.

Ethical approval for the study was given by the Semmelweis University Institutional Review Board (SE-TUKEB); the number of the ethical approval is SE-TUKEB No.119/2013 (Date of approval: 24 June 2013).

Informed Consent Informed consent was obtained from all individual participants included in the study.

Open Access This article is distributed under the terms of the Creative Commons Attribution 4.0 International License (http:// creativecommons.org/licenses/by/4.0/), which permits unrestricted use, distribution, and reproduction in any medium, provided you give appropriate credit to the original author(s) and the source, provide a link to the Creative Commons license, and indicate if changes were made.

\section{References}

1. International Atomic Energy Agency (2013) Standard operation procedures for PET/CT: a practical approach for use in adult oncology. IAEA Human Health Series No.26, Vienna

2. Groheux D, Espie M, Giacchetti S, Hindie E (2013) Performance of FDG PET/CT in the clinical management of breast cancer. Radiology 266:388-405

3. Kajáry K, Tökés T, Dank M, Kulka J, Szakáll S Jr, Lengyel Z (2015) Correlation of the value of $18 \mathrm{~F}-\mathrm{FDG}$ uptake, described by SUVmax, SUVavg, metabolic tumour volume and total lesion glycolysis, to clinicopathological prognostic factors and biological subtypes in breast cancer. Nucl Med Comm 36:28-37

4. Huang SC (2000) Anatomy of SUV. Nucl Med Biol 27:643-646

5. Morris ED, Endres CJ, Schmidt KC, Christian BT, Muic RJ, Fisher RE (2004) Kinetic modeling in positron emission tomography. In: Wernick MN, Aarsvold JN (eds) Emission tomography. The fundamentals of PET and SPECT. Elsevier Academic Press, San Diego, pp 499-540

6. Kelloff GJ, Hoffman JM, Johnson B, Scher HI, Siegel BA, Cheng EY et al (2005) Progress and promise of FDG-PET imaging for cancer patient management and oncologic drug development. Clin Cancer Res 11:2785-2808

7. Curigliano G, Burstein HJ, Winer EP, Gnant M, Dubsky P, Loibl S et al (2017) De-escalating and escalating treatments for early-stage breast cancer: the St Gallen international expert consensus 
conference on the primary therapy of early breast Cancer 2017. Ann Oncol 28:1700-1712

8. Salgado R, Denkert C, Demaria S, Sirtaine N, Klauschen F, Pruneri $G$ et al (2015) The evaluation of tumor-infiltrating lymphocytes (TILs) in breast cancer: recommendations by an International TILsWorking Group 2014. Ann Oncol 26:259-271

9. Tökés T, Kajáry K, Dank M, Lengyel Z (2018) Motion correction during dynamic FDG-PET/CT studies performed in the staging of breast cancer. Quarterly Journal of Nuclear Medicine and Molecular Imaging. https://doi.org/10.23736/S1824-4785.19. 03114-5

10. Soret M, Bacharach SL, Buvat I (2007) Partial-volume effect in PET tumor imaging. J Nucl Med 48:932-945

11. Brierley JD, Gospodarowicz MK, Wittekind C, editors in chief (2017) TNM classification of malignant tumours. Eighth Edition. Oxford: Wiley

12. Allred DC, Harvey JM, Berardo M, Clark GM (1998) Prognostic and predictive factors in breast cancer by immunohistochemical analysis. Mod Pathol 11:155-168

13. Wolff AC, Hammond ME, Schwartz JN, Hagerty KL, Allred DC, Cote RJ et al (2007) American Society of Clinical Oncology/ College of American Pathologists guideline recommendations for human epidermal growth factor receptor 2 testing in breast cancer. $\mathrm{J}$ Clin Oncol 25:118-145

14. Wolff AC, Hammond ME, Hicks DG, Dowsett M, McShane LM, Allison KH et al (2013) Recommendations for human epidermal growth factor receptor 2 testing in breast cancer: American Society of Clinical Oncology/College of American Pathologists clinical practice guideline update. J Clin Oncol 31:3997-4013

15. Dowsett M, Nielsen TO, A'Hern R, Bartlett J, Coombes RC, Cuzick $\mathrm{J}$ et al (2011) Assessment of Ki67 in breast cancer: recommendations from the International Ki67 in Breast Cancer working group. J Natl Cancer Inst 103:1656-1664

16. Pathmanathan N, Balleine RL (2013) Ki67 and proliferation in breast cancer. J Clin Pathol 66:512-516

17. Tokes AM, Szasz AM, Geszti F, Lukacs LV, Kenessey I, Turanyi E et al (2015) Expression of proliferation markers Ki67, cyclin A, geminin and aurora-kinase $\mathrm{A}$ in primary breast carcinomas and corresponding distant metastases. J Clin Pathol 68:274-282

18. Shankar LK, Hoffman JM, Bacharach S, Graham MM, Karp J, Lammertsma AA et al (2006) Consensus recommendations for the use of 18F-FDG PET as an indicator of therapeutic response in patients in National Cancer Institute Trials. J Nucl Med 47: 1059-1066

19. Boellaard R, O'Doherty MJ, Weber WA, Mottaghy FM, Lonsdale MN, Stroobants SG et al (2010) FDG PET and PET/CT: EANM procedure guidelines for tumour PET imaging: version 1.0. Eur J Nucl Med Mol Imaging 37:181-200

20. Mankoff DA, Dunnwald LK, Gralow JR, Ellis GK, Charlop A, Lawton TJ et al (2002) Blood flow and metabolism in locally advanced breast cancer: relationship to response to therapy. $\mathrm{J}$ Nucl Med 43:500-509

21. Dunnwald LK, Doot RK, Specht JM, Gralow JR, Ellis GK, Livingston RB et al (2011) PET tumor metabolism in locally advanced breast cancer patients undergoing neoadjuvant chemotherapy: value of static versus kinetic measures of fluorodeoxyglucose uptake. Clin Cancer Res 17:2400-2409

22. Doot RK, Dunnwald LK, Schubert EK, Muzi M, Peterson LM, Kinahan PE et al (2007) Dynamic and static approaches to quantifying $18 \mathrm{~F}-\mathrm{FDG}$ uptake for measuring cancer response to therapy, including the effect of granulocyte CSF. J Nucl Med 48:920-925

23. Partridge SC, Vanantwerp RK, Doot RK, Chai X, Kurland BF, Eby PR et al (2010) Association between serial dynamic contrastenhanced MRI and dynamic 18F-FDG PET measures in patients undergoing neoadjuvant chemotherapy for locally advanced breast cancer. J Magn Reson Imaging 32:1124-1131

24. Tseng J, Dunnwald LK, Schubert EK, Link JM, Minoshima S, Muzi M et al (2004) 18F-FDG kinetics in locally advanced breast cancer: correlation with tumor blood flow and changes in response to neoadjuvant chemotherapy. J Nucl Med 45:1829-1837

25. Dunnwald LK, Gralow JR, Ellis GK, Livingston RB, Linden HM, Specht JM et al (2008) Tumor metabolism and blood flow changes by positron emission tomography: relation to survival in patients treated with neoadjuvant chemotherapy for locally advanced breast cancer. J Clin Oncol 26:4449-4457

26. Eby PR, Partridge SC, White SW, Doot RK, Dunnwald LK, Schubert EK et al (2008) Metabolic and vascular features of dynamic contrast-enhanced breast magnetic resonance imaging and (15)O-water positron emission tomography blood flow in breast cancer. Acad Radio 15:1246-1254

27. Wangerin KA, Muzi M, Peterson LM, Linden HM, Novakova A, Mankoff DA et al (2017) A virtual clinical trial comparing static versus dynamic PET imaging in measuring response to breast cancer therapy. Phys Med Biol 62:3639-3655

28. Lee S, Choi S, Kim SY, Yun MJ, Kim HI (2017) Potential utility of FDG PET-CT as a non-invasive tool for monitoring local immune responses. J Gastric Cancer 17:384-393

29. Lopci E, Toschi L, Grizzi F, Rahal D, Olivari L, Castino GF et al (2016) Correlation of metabolic information on FDG-PET with tissue expression of immune markers in patients with non-small cell lung cancer (NSCLC) who are candidates for upfront surgery. Eur J Nucl Med Mol Imaging 43:1954-1961

30. Fujii T, Yanai K, Tokuda S, Nakazawa Y, Kurozumi S, Obayashi S et al (2018) Relationship between FDG uptake and neutrophil/ lymphocyte ratio in patients with invasive ductal breast cancer. Anticancer Res 38:4927-4931

Publisher's Note Springer Nature remains neutral with regard to jurisdictional claims in published maps and institutional affiliations. 